\title{
Vocal cord paralysis and oesophago-broncho-aortic fistula complicating foreign body-induced oesophageal perforation
}

\author{
A.S. Taha, I. Nakshabendi and R.I. Russell \\ Gastroenterology Unit, Royal Infirmary, Glasgow G31 2ER, UK
}

\begin{abstract}
Summary: A 61 year old man died after presenting with a $24 \mathrm{~h}$ history of haematemesis and haemoptysis, and one year history of hoarseness of voice. Post-mortem examination showed a dental plate eroding through the mid-oesophagus into a bronchus and into the descending arch of the aorta, with scarring suggestive of old perforation. An organized haematoma also involved the left recurrent laryngeal nerve. Vocal cord paralysis may be a manifestation of foreign body-induced oesophageal perforation, which can lead to death from an oesophago-broncho-aortic fistula. Both complications of oesophageal perforation from a foreign body have not to our knowledge been previously reported.
\end{abstract}

\section{Introduction}

Foreign body-induced oesophageal perforation is a potentially lethal condition which requires early identification and prompt intervention..$^{1,2}$ The diagnosis in the majority of cases is made within 48 hours of the ingestion of a foreign body, with the development of dysphagia, chest pain, haematemesis, and certain radiographic findings. ${ }^{1-3} \mathrm{We}$ report a case which presented 18 months after swallowing a piece of denture, and was complicated by vocal cord paralysis and oesophago-bronchoaortic fistula.

\section{Case report}

A 61 year old man was admitted with a 24 hour history of recurrent episodes of haematemesis and haemoptysis. He also had hoarseness of voice, which was diagnosed as being idiopathic in origin in another hospital about a year prior to his current admission. He had no dysphagia, heartburn, chest or abdominal pain, and no weight loss. On physical examination, he was sweating, his temperature was $35^{\circ} \mathrm{C}$, pulse rate 130 beats $/ \mathrm{min}$, and blood pressure $110 / 60 \mathrm{mmHg}$. The rest of his examination was unremarkable apart from hoarseness of voice.

His haemoglobin was $8.5 \mathrm{~g} / \mathrm{dl}$, urea $12.2 \mathrm{mmol} / 1$, creatinine $80 \mu \mathrm{mol} / 1$, and chest radiograph was

Correspondence: A.S. Taha, M.D., M.R.C.P. Accepted: 1 November 1991 normal. Resuscitation was started with intravenous fluids and blood transfusion. Upper gastrointestinal endoscopy showed a mid-oesophageal lesion measuring $5 \mathrm{~cm}$ in diameter, with irregular and necrotic edges and a blood clot at the centre. No active bleeding was observed. This gave the impression of a necrotic oesophageal carcinoma. No ulcers were seen in the stomach or duodenum.

Within 15 minutes of endoscopy the patient had further haemoptysis and haematemesis, became shocked, and subsequently died. Post-mortem examination showed a piece of denture impacted in the mid-oesophagus and eroding into a bronchus and the descending aorta, thus creating an oesophago-broncho-aortic fistula. There was scarring and inflammatory tissue around the site, indicating that the foreign body had been impacted in the oesophagus for a long period of time. The left recurrent laryngeal nerve was involved by an organized haematoma. There was no evidence of malignancy in the oesophagus, larynx or lungs.

Previous case records were later obtained and showed that the patient had presented 18 months before his death with one episode of coughing and choking while he was eating. He then vomited into the toilet and noticed that his dental plate was missing. He was not sure whether he swallowed his dental plate or lost it in the toilet. The patient was observed for 24 hours and discharged without any other complaints. Six months later, a year before his death, he complained of hoarseness of voice. Laryngoscopy showed left vocal cord paralysis and 
chest radiograph was normal. His condition remained stable until his final presentation.

\section{Discussion}

This case report suggests that an apparently obscure vocal cord paralysis might be secondary to previous oesophageal perforation by a foreign body such as a dental plate. The unusual aspects of this case relate to the nature of, and the delayed presentation of the complications of oesophageal perforation: 6 months for vocal cord paralysis and 18 months for the oesophago-broncho-aortic fistula. Although an aorto-oesophageal fistula is a well-recognized complication of oesophageal perforation, a fistula connecting the aorta and oesophagus to the bronchial tree has not been

\section{References}

1. Nandi, P. \& Ong, G.B. Foreign body in the oesophagus: review of 2,394 cases. Br J Surg 1978, 65: 5-9.

2. Branks, J.G. \& Bancewicz, J. Perforation of the oesophagus: experience in a general hospital. Br J Surg 1981, 68: 580-584.

3. Appleton, D.S., Sandrasagra, F.A. \& Flower, C.D. Perforated oesophagus: review of twenty-eight consecutive cases. Clin Radiol 1979, 30: 493-497. previously described, to our knowledge, and nor has vocal cord paralysis. A recent report has suggested that aorto-oesophageal fistulae can be treated by surgery. ${ }^{4}$ However, the vast majority of cases reported, like our patient, resulted in death. ${ }^{1}$ At endoscopy, the mid-oesophagus of our patient had a necrotic lesion with a central blood clot suggestive of malignancy. We believe that such features should also be considered as indicative of established oesophageal perforation when such possibility is suspected.

This case report illustrates some rare, but potentially treatable, complications of foreign bodyinduced oesphageal perforation. Despite the fact that early diagnosis and prompt treatment are of critical importance, it should be appreciated that delayed presentation can occur.

4. Schiessel, R., Rath, T. \& Kretschmer, G. Giant ulcer of the oesophagus with erosion of the aorta - successful treatment with aortic suture secured by an omental graft and oesophagectomy. Br J Surg 1985, 72: 650. 